\title{
University of Florida Potato Variety Trials Spotlight: 'LaChipper'1
}

\author{
Mario H. M. L. Andrade, Rodrick Z. Mwatuwa, Christian T. Christensen, Pam Solano, and \\ Lincoln Zotarelli²
}

There are several potato varieties available in the market today. Most of them have been bred or developed in production regions other than Florida. The University of Florida Potato Variety Evaluation Program screens new germplasm from public and private breeding programs and identifies the most promising cultivars for commercial potential considering broad adaptability to Florida climate and conditions and market purpose: processing, freshmarket and specialty-type varieties. Over the years, the UF/ IFAS Potato Variety Program has become an important reference to vegetable growers, seed producers, processors, crop insurance agencies, and brokers looking for alternative potato varieties to explore different markets, improved characteristics, and yield. This UF/Potato Variety Trials Spotlight presents a summary of the field evaluation of tuber yield and quality performance of the potato variety 'LaChipper' cultivated in Florida.

\section{General Comments}

'LaChipper' is a potato variety that is commonly grown for the fresh potato market in Florida. Under different growing conditions, 'LaChipper' is suited for chip processing. It was selected from progeny of a cross between Green Mountain and Cayuga and tested under the pedigree L91-78. It was released by the Department of Horticulture and Landscape Architecture, Louisiana Agricultural Experiment Station,
Baton Rouge, LA in October of 1962. 'LaChipper' demonstrates high yield and good tuber characteristics compared to the commercial standard 'Atlantic'. Tuber production and quality results provided in this spotlight are from Florida Potato Variety Trials conducted at the UF/IFAS Hastings Agricultural Extension Center between 1998 and 2016.

\section{General Characteristics}

'LaChipper' tubers are moderately smooth with a round to oblong shape (Figure 1). According to Florida's rating codes for potato tuber characteristics (Table 1), the tubers have a fair to good appearance with buff skin color, white flesh, and deep to intermediate eye depth. On average, the marketable yield is $256 \mathrm{cwt} /$ acre, approximately $12 \%$ less than the commercial standard 'Atlantic', with $83 \%$ of the tubers produced found between A1 and A3 tuber size classifications (Table 2).

\section{Season Length and Growth}

'LaChipper' is a medium-maturing cultivar under Florida growing conditions. Season length is 95 days on average from planting to harvest. This depends on weather conditions during the growing season. The plants should be harvested two to three weeks after vine kill to improve tuber maturation and skin set. Potatoes with proper skin

1. This document is HS1297, one of a series of the Horticultural Sciences Department, UF/IFAS Extension. Original publication date May 2017. Revised April 2020. Visit the EDIS website at https://edis.ifas.ufl.edu.

2. Mario H. M. L. Andrade, research scholar; Rodrick Z. Mwatuwa, research assistant; Christian T. Christensen, regional specialized agent II, director, UF/ IFAS Hastings Agricultural Extension Center; Pam Solano, biological scientist; Lincoln Zotarelli, assistant professor; Horticultural Sciences Department, UF/IFAS Extension, Gainesville, FL 32611.

The Institute of Food and Agricultural Sciences (IFAS) is an Equal Opportunity Institution authorized to provide research, educational information and other services

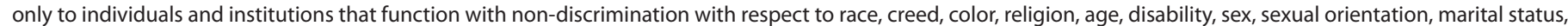

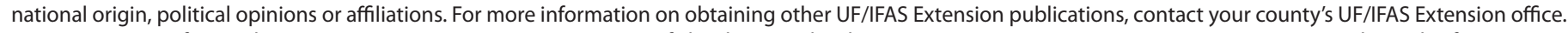
U.S. Department of Agriculture, UF/IFAS Extension Service, University of Florida, IFAS, Florida A \& M University Cooperative Extension Program, and Boards of County Commissioners Cooperating. Nick T. Place, dean for UF/IFAS Extension. 
set maintain better skin color, lose less weight in storage, and are more resistant to bruising and soft rot. For more information about vine killing on potatoes, see Potato Vine Killing or Desiccation (Zotarelli et al. 2016). Late in the season, tuber size should be checked regularly to harvest tubers with desirable marketable size. Soil moisture should be managed late in the season to avoid high soil moisture conditions that cause enlarged lenticels and delayed skin set.

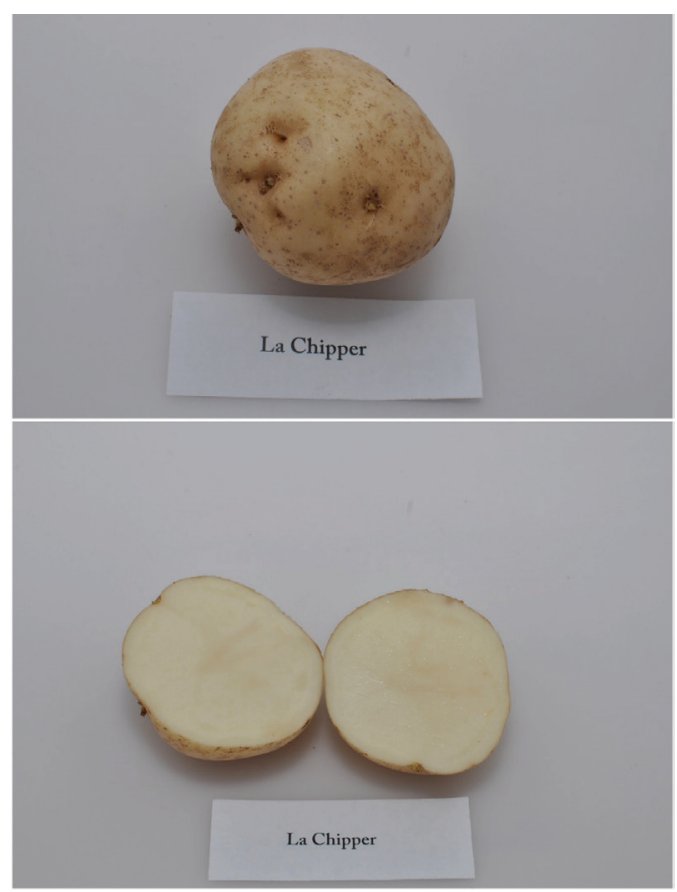

Figure 1. Typical tuber and internal flesh color of 'LaChipper' potato variety.

Credits: Lincoln Zotarelli, UF/IFAS

\section{Diseases}

'LaChipper' demonstrates low incidence of hollow heart (1\%) and corky ring spot (2\%) under Florida conditions (Table 3). It has some resistance to late blight (Phytophthora infestans) and is moderately susceptible to common scab (Streptomyces scabies). Exposure to air pollution can result in defoliation and reduction in tuber yield.

The standard UF/IFAS Extension recommended disease and weed control program described under Potato Production (Chapter 14 of the Vegetable Production Handbook for Florida, http://edis.ifas.ufl.edu/cv131) should be followed.

\section{Fertilization}

UF/IFAS trial plots are normally fertilized with 200 to 230 $\mathrm{lb} / \mathrm{ac}$ of $\mathrm{N}$. The first application of $100 \mathrm{lb} / \mathrm{ac}$ of $\mathrm{N}$ (granular) is typically incorporated in the bed prior to planting, followed by one or two side-dress fertilizer applications at emergence and/or at tuber initiation. Phosphorus and potassium applications follow UF/IFAS guidelines described in Liu et al. (2020) and normally range between 45 to $100 \mathrm{lb} / \mathrm{ac}$ of $\mathrm{P}_{2} \mathrm{O}_{5}$ and 170 to $235 \mathrm{lb} / \mathrm{ac}$ of $\mathrm{K}_{2} \mathrm{O}$.

\section{Planting}

A seed piece of 2.5 to $3 \mathrm{oz}$ is recommended for planting. The crop should be planted with 40 inches between rows and 8 inches between plants, at 3 to 4 inches deep. A seed rate of 2,000 to $3,000 \mathrm{lb} /$ acre of seed is expected.

\section{Other Information}

For additional information on cultivation and weed and disease management, see the Potato Production chapter of the Vegetable Production Handbook, available at http://edis. ifas.ufl.edu/cv131.

\section{References}

Hutchinson, C. M., J. M. White, D. M., Gergela, P. A. Solano, K. G. Haynes, R. Wenrich, and C. S. Lippi. 2003. "Performance of chip processing potato varieties in northeastern Florida." HortTechnology 13 (4): 706-711. https:// doi.org/10.21273/HORTTECH.13.4.0706

Liu, G., E. H. Simonne, K. T. Morgan, G. J. Hochmuth, S. Agehara, and R. Mylavarapu. 2020. Chapter 2. Fertilizer Management for Vegetable Production in Florida. Vegetable Production Handbook for Florida, 2020-2021 Edition. CV296. Gainesville: University of Florida Institute of Food and Agricultural Sciences. http://edis.ifas.ufl.edu/cv296

Miller, Julian C., James F. Fontenot, and W. A. Young. 1963. "LaRouge and LaChipper two new potato varieties released by Louisiana." American Potato Journal 40 (4): 130-132. https://doi.org/10.21273/HORTTECH.13.4.0706

Sisson, J. A., and G. A. Porter. 2002. "Performance evaluations of potato clones and varieties in the northeastern states-1999." Maine Agr. For. Expt. Sta., Misc. Publ. 751.

Zotarelli, L., P. J. Dittmar, P. D. Roberts, J. Desaegar, and B. Wells.. 2020. Chapter 14. Potato Production. Vegetable Production Handbook for Florida, 2020-2011 Edition. HS733. Gainesville: University of Florida Institute of Food and Agricultural Sciences. http://edis.ifas.ufl.edu/cv131

Zotarelli, L., S. Sargent, P. Dittmar, and M. Makani. 2016. Potato Vine Killing or Desiccation. HS181. Gainesville: University of Florida Institute of Food and Agricultural Sciences. http://edis.ifas.ufl.edu/hs181 
Table 1. Summary of production statistics and specific gravity of 'LaChipper' potato variety grown at the UF/IFAS Hastings Agricultural Extension Center, Hastings, FL from 1998 to 2016.

\begin{tabular}{|c|c|c|c|c|c|c|c|c|c|c|c|c|c|}
\hline \multirow[t]{2}{*}{ Year } & \multirow{2}{*}{$\begin{array}{l}\text { Total } \\
\text { Yield }\end{array}$} & \multirow{2}{*}{$\begin{array}{c}\text { Marketable } \\
\text { Yield }^{1}\end{array}$} & \multirow{2}{*}{$\begin{array}{l}\% \text { of } \\
\text { STD }\end{array}$} & \multirow[t]{2}{*}{ Standard } & \multicolumn{6}{|c|}{ Size Class (Distribution by class $\%)^{2}$} & \multicolumn{2}{|c|}{ Range \% } & \multirow{2}{*}{$\begin{array}{l}\text { Specific } \\
\text { Gravity }\end{array}$} \\
\hline & & & & & C & B & A1 & A2 & A3 & A4 & $A 1$ to $A 3$ & Culls & \\
\hline 1998 & 391 & 333 & 96 & Atlantic & 12 & 4 & 30 & 40 & 16 & 0 & 86 & 12 & 1.068 \\
\hline 1999 & 375 & 334 & 88 & Atlantic & 8 & 3 & 64 & 25 & 1 & 0 & 89 & 8 & 1.063 \\
\hline 2000 & 281 & 245 & 72 & Atlantic & 0 & 17 & 32 & 27 & 26 & 0 & 87 & 8 & 1.070 \\
\hline 2001 & 297 & 272 & 81 & Atlantic & 0 & 4 & 49 & 38 & 9 & 0 & 96 & 5 & 1.072 \\
\hline 2002 & 324 & 302 & 92 & Atlantic & 0 & 4 & 43 & 44 & 9 & 0 & 96 & 3 & 1.068 \\
\hline 2003 & 436 & 321 & $*$ & Atlantic & 12 & 9 & 46 & 18 & 15 & 0 & 79 & 7 & 1.068 \\
\hline 2004 & 344 & 234 & 101 & Atlantic & 17 & 11 & 57 & 13 & 2 & 0 & 72 & 6 & 1.074 \\
\hline 2005 & 302 & 246 & 79 & Atlantic & 1 & 11 & 77 & 10 & 1 & 0 & 88 & 7 & 1.071 \\
\hline 2006 & 398 & 337 & 99 & Atlantic & 1 & 10 & 70 & 14 & 4 & 0 & 89 & 5 & 1.071 \\
\hline 2007 & 374 & 310 & 85 & Atlantic & 2 & 12 & 69 & 14 & 3 & 0 & 87 & 5 & 1.070 \\
\hline 2008 & 342 & 240 & 111 & Atlantic & 3 & 24 & 67 & 6 & 1 & 0 & 73 & 4 & 1.073 \\
\hline 2009 & 329 & 248 & 124 & Atlantic & 3 & 16 & 64 & 13 & 4 & 0 & 82 & 11 & 1.064 \\
\hline 2010 & 367 & 282 & 102 & Atlantic & 3 & 17 & 59 & 16 & 5 & 0 & 80 & 4 & 1.066 \\
\hline 2011 & 349 & 294 & 111 & Atlantic & 2 & 8 & 67 & 12 & 10 & 0 & 89 & 6 & 1.067 \\
\hline 2012 & 353 & 274 & 84 & Atlantic & 2 & 6 & 56 & 21 & 15 & 0 & 92 & 15 & 1.067 \\
\hline 2013 & 317 & 262 & 91 & Atlantic & 3 & 10 & 64 & 11 & 11 & 0 & 86 & 6 & 1.067 \\
\hline 2014 & 221 & 108 & 47 & Atlantic & 6 & 32 & 62 & 0 & 0 & 0 & 62 & 25 & 1.061 \\
\hline 2015 & 162 & 109 & 49 & Atlantic & 3 & 25 & 71 & 0 & 0 & 0 & 71 & 5 & 1.079 \\
\hline 2016 & 178 & 112 & 77 & Atlantic & 4 & 15 & 71 & 5 & 6 & 0 & 81 & 22 & 1.063 \\
\hline Average & 323 & 256 & 88 & & 4 & 13 & 59 & 17 & 7 & 0 & 83 & 9 & 1.069 \\
\hline \multicolumn{14}{|c|}{$\begin{array}{l}{ }^{1} \text { Marketable yield: Sum of size classes } A 1 \text { to } A 3 \text {. } \\
\text { 2Size classes: } C=0.5 \text { to } 1.5 \text { inches, } B=1.5 \text { to } 17 / 8 \text { inches, } A 1=17 / 8 \text { to } 2.5 \text { inches, } A 2=2.5 \text { to } 3.25 \text { inches, } A 3=3.25 \text { to } 4 \text { inches, } A 4>4 \text { inches; } \\
\text { Size distribution by class: Class (wt)/(Total Yield [wt] - culls [wt]) } \\
{ }^{*} \text { Missing data }\end{array}$} \\
\hline
\end{tabular}

Table 2. Florida rating codes for potato vine maturity and tuber characteristics.

\begin{tabular}{|c|c|c|c|c|c|c|c|}
\hline \multicolumn{8}{|c|}{ Tuber Characteristics ${ }^{1}$} \\
\hline Rating Code & Vine Maturity & $\begin{array}{c}\text { Internal } \\
\text { Flesh Color }\end{array}$ & Skin Color & Skin Texture & Tuber Shape & Eye Depth & $\begin{array}{c}\text { Overall Tuber } \\
\text { Appearance }\end{array}$ \\
\hline 1 & dead & white & purple & partial russet & round & very deep & very poor \\
\hline 2 & +- & cream & red & heavy russet & mostly round & +- & +- \\
\hline 3 & yellow and dying & light yellow & pink & moderate russet & round to oblong & deep & poor \\
\hline 4 & +- & $\begin{array}{l}\text { medium } \\
\text { yellow }\end{array}$ & dark brown & light russet & mostly oblong & +- & +- \\
\hline 5 & $\begin{array}{c}\text { moderately } \\
\text { senesced }\end{array}$ & dark yellow & brown & netted & oblong & intermediate & fair \\
\hline 6 & +- & pink & $\tan$ & slightly netted & oblong to long & +- & +- \\
\hline 7 & starting to senesce & red & buff & $\begin{array}{l}\text { moderately } \\
\text { smooth }\end{array}$ & mostly long & shallow & good \\
\hline 8 & +- & blue & white & smooth & long & +- & +- \\
\hline 9 & $\begin{array}{l}\text { green and } \\
\text { vigorous }\end{array}$ & purple & cream & very smooth & cylindrical & very shallow & Excellent \\
\hline
\end{tabular}


Table 3. Vine maturity, tuber characteristics, and internal tuber defects of 'LaChipper' potato variety grown at the UF/IFAS Hastings Agricultural Extension Center, Hastings, FL from 1998 to 2016.

\begin{tabular}{|c|c|c|c|c|c|c|c|c|c|c|c|}
\hline \multirow[t]{2}{*}{ Year } & \multirow{2}{*}{$\begin{array}{c}\text { Vine } \\
\text { Maturity }\end{array}$} & \multicolumn{6}{|c|}{ Tuber Characteristics ${ }^{1}$} & \multicolumn{4}{|c|}{ Internal Defects ${ }^{2}$} \\
\hline & & $\begin{array}{l}\text { Internal } \\
\text { Flesh Color }\end{array}$ & Skin Color & Skin Texture & Tuber Shape & Eye Depth & $\begin{array}{c}\text { Overall } \\
\text { Appearance }\end{array}$ & HH & BR & CRS & IHN \\
\hline 1998 & $*$ & $*$ & 8 & 8 & 3 & 5 & 6 & $*$ & $*$ & $*$ & $*$ \\
\hline 1999 & $*$ & $*$ & 8 & 6 & 2 & 4 & 6 & 0 & 0 & 0 & 0 \\
\hline 2000 & $*$ & $*$ & 8 & 8 & 3 & 5 & 6 & 0 & 0 & 0 & 0 \\
\hline 2001 & 1 & 1 & 7 & 7 & 3 & 5 & 6 & 0 & 0 & 2 & 0 \\
\hline 2002 & 3 & 1 & 8 & 7 & 3 & 6 & 7 & 0 & 0 & 10 & 1 \\
\hline 2003 & 4 & 1 & 8 & 7 & 2 & 6 & 6 & 3 & 0 & 0 & 0 \\
\hline 2004 & 6 & 3 & 8 & 7 & 2 & 5 & 6 & 1 & 0 & 0 & 0 \\
\hline 2005 & 6 & 1 & 9 & 8 & 3 & 5 & 6 & 0 & 0 & 0 & 0 \\
\hline 2006 & 7 & 1 & 8 & 8 & 4 & 6 & 7 & 0 & 0 & 0 & 0 \\
\hline 2007 & 6 & 1 & 7 & 7 & 3 & 3 & 7 & 1 & 0 & 0 & 0 \\
\hline 2008 & 6 & 2 & 8 & 8 & 2 & 6 & 6 & 0 & 0 & 0 & 0 \\
\hline 2009 & 4 & 1 & 6 & 7 & 3 & 3 & 7 & 0 & 0 & 0 & 0 \\
\hline 2010 & 7 & 1 & 8 & 8 & 4 & 3 & 7 & 1 & 0 & 0 & 0 \\
\hline 2011 & 3 & * & * & $*$ & $*$ & $*$ & 6 & 0 & 0 & 28 & 0 \\
\hline 2012 & 5 & $*$ & $*$ & * & $*$ & $*$ & 6 & 0 & 0 & 0 & 0 \\
\hline 2013 & 6 & $*$ & $*$ & $*$ & $*$ & $*$ & 6 & 5 & 0 & 0 & 0 \\
\hline 2014 & 2 & 1 & 8 & 7 & 3 & 4 & 5 & 2 & 0 & 0 & 0 \\
\hline 2015 & 5 & 0 & 0 & 0 & 0 & 0 & 7 & 0 & 0 & 0 & 0 \\
\hline 2016 & 6 & 0 & 3 & 3 & 1 & 2 & 7 & 0 & 0 & 1 & 0 \\
\hline Average & 5 & 1 & 7 & 7 & 3 & 4 & 6 & 1 & 0 & 2 & 0 \\
\hline
\end{tabular}

